
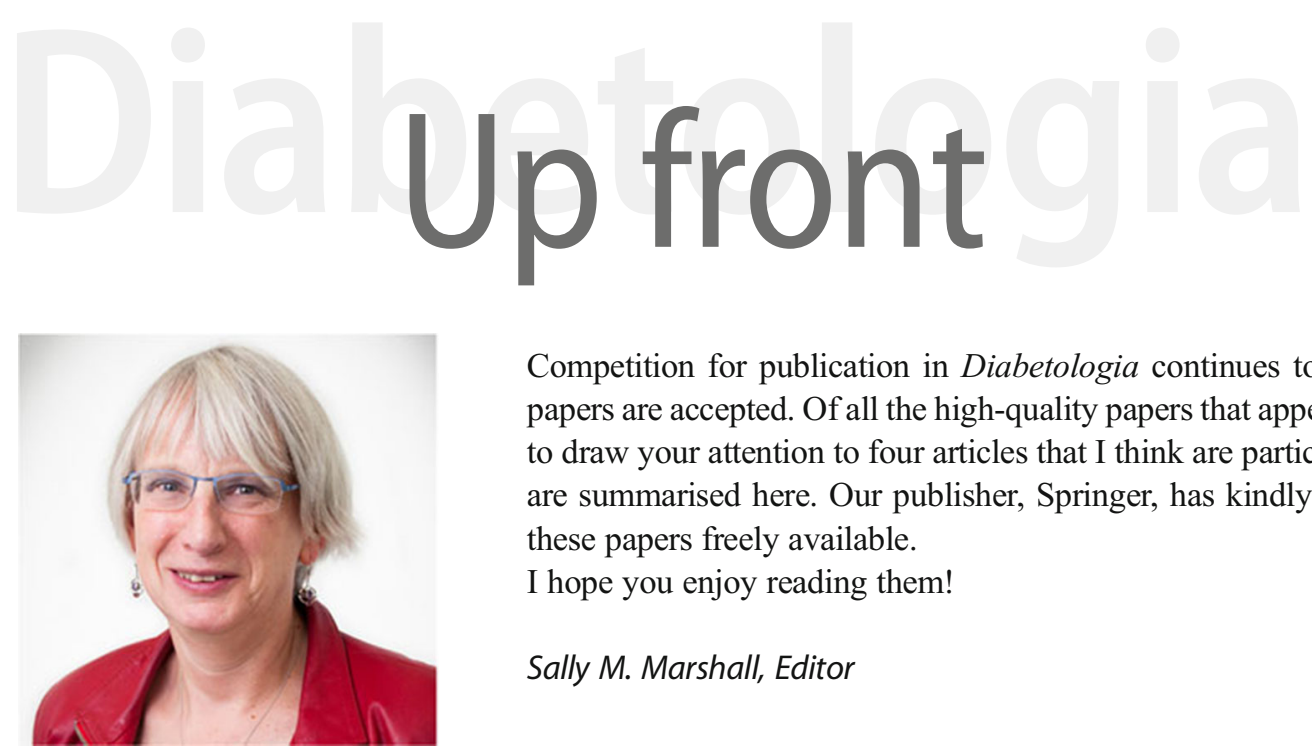

Competition for publication in Diabetologia continues to grow, and less than $20 \%$ of papers are accepted. Of all the high-quality papers that appear in this month's issue I want to draw your attention to four articles that I think are particularly interesting. The articles are summarised here. Our publisher, Springer, has kindly made the full text of each of these papers freely available.

I hope you enjoy reading them!

Sally M. Marshall, Editor

\section{Obesity, unfavourable lifestyle and genetic risk of type 2 diabetes: a case-cohort study}

Theresia M. Schnurr, Hermina Jakupović, Germán D. Carrasquilla, Lars Ängquist, Niels Grarup, Thorkild I. A. Sørensen, Anne Tjønneland, Kim Overvad, Oluf Pedersen, Torben Hansen, Tuomas O. Kilpeläinen

Type 2 diabetes is a common disease with a rapidly increasing global prevalence that has been largely attributed to the ongoing pandemic of obesity and a sedentary lifestyle. However, it is unclear whether the effects of obesity and unfavourable lifestyle on diabetes risk may vary depending on genetic variation. In this issue, Schnurr, Jakupović et al (https://doi.org/10.1007/s00125020-05140-5) investigated whether the associations of obesity and unfavourable lifestyle with increased risk of type 2 diabetes are accentuated by genetic predisposition. Analysing data from 4729 individuals who developed incident type 2 diabetes and a randomly selected cohort sample of 5402 individuals, the authors found that obesity and unfavourable lifestyle are associated with increased diabetes risk irrespective of genetic predisposition. The authors conclude that weight management by healthy lifestyle should be recommended as a prevention strategy for type 2 diabetes, regardless of genetic predisposition.
The pore-forming subunit MCU of the mitochondrial $\mathrm{Ca}^{2+}$ uniporter is required for normal glucose-stimulated insulin secretion in vitro and in vivo in mice

Eleni Georgiadou, Elizabeth Haythorne, Matthew T. Dickerson, Livia Lopez-Noriega, Timothy J. Pullen, Gabriela da Silva Xavier, Samuel P. X. Davis, Aida Martinez-Sanchez, Francesca Semplici, Rosario Rizzuto, James A. McGinty, Paul M. French, Matthew C. Cane, David A. Jacobson, Isabelle Leclerc, Guy A. Rutter

Impaired $\mathrm{Ca}^{2+}$ signalling in the pancreatic beta cell contributes to deficiencies in insulin secretion in type 2 diabetes. Intramitochondrial oxidative metabolism is thought to be stimulated by increases in cytosolic $\mathrm{Ca}^{2+}$; the mitochondrial uniporter (MCU) complex and its associated regulatory proteins are proposed to be the main driving force of $\mathrm{Ca}^{2+}$ entry into mitochondria. In this issue, Georgiadou et al (https://doi.org/10.1007/s00125-020-05148-x) tested this hypothesis by selectively eliminating $\mathrm{Mcu}$ expression in the beta cells of mice. Mitochondrial $\mathrm{Ca}^{2+}$ uptake, ATP production, and insulin secretion in response to glucose stimulation were all substantially impaired, in vitro, in the absence of Mcu. However, the in vivo phenotype of mice lacking Mcu was more complex, with glucose-stimulated insulin release varying depending on test conditions (e.g. time after glucose injection, method of glucose administration and 
age of animals). The authors conclude that these findings suggest that altered mitochondrial $\mathrm{Ca}^{2+}$ uptake may contribute to defective insulin secretion in some forms of diabetes, and highlight MCU as a potential therapeutic target.

\section{Insulin sensitivity depends on the route of glucose administration}

Geltrude Mingrone, Simona Panunzi, Andrea De Gaetano, Sofie Ahlin, Valerio Spuntarelli, Isabel Bondia-Pons, Chiara Barbieri, Esmeralda Capristo, Amalia Gastaldelli, John J Nolan

Bariatric surgery results in a rapid improvement in insulin resistance long before weight loss, focusing attention on the role of the small intestine in insulin resistance. In this issue, Mingrone et al (https://doi.org/10.1007/s00125-020-05157w) compared glucose metabolism in obese participants who were administered oral glucose and, after 7-10 days, i.v. glucose (plasma glucose during i.v. administration was matched to plasma glucose during oral challenge). They also analysed the differences in insulin effect on proteolysis, ketogenesis and lipolysis. The authors found that the oral route led to increased insulin secretion and a complementary $40 \%$ reduction in glucose clearance, as compared with the i.v. route. The efficacy of insulin to reduce lipolysis and proteolysis also decreased after oral glucose administration. The authors suggest that an unknown mechanism, triggered by the presence of glucose in the intestinal lumen, counterbalances the effect of incretins (gut hormones that stimulate insulin secretion) by limiting the effect of insulin that has been released in response to glucose. They conclude that these findings indicate that insulin sensitivity depends on the route of glucose administration and that (as yet unknown) intestinal factors are crucial to these underlying metabolic processes.

\section{Decreased pancreatic acinar cell number in type 1 diabetes}

Jordan J. Wright, Diane C. Saunders, Chunhua Dai, Greg Poffenberger, Brynn Cairns, David V. Serreze, David M. Harlan, Rita Bottino, Marcela Brissova, Alvin C. Powers

Individuals with type 1 diabetes have a reduced total pancreas size; however, the cellular and tissue changes responsible for this size reduction are not well characterised. In this issue, Wright et al (https://doi.org/10.1007/s00125-020-05155-y) report that pancreases from donors with type 1 diabetes were approximately $45 \%$ smaller than those from non-diabetic donors. They also had fewer total acinar cells and, as expected, a lower beta cell mass. Acinar cell size was similar between pancreases from diabetic and non-diabetic donors, regardless of location within pancreatic lobes or proximity to islets. Compared with non-diabetic donors, pancreases from donors with type 1 diabetes also had increased fibrosis. Based on the analysis of size and cell composition of pancreases from donors with a wide range of type 1 diabetes duration, the authors speculate that the decline in acinar cell number begins very early in disease progression and that type 1 diabetes pathogenesis involves both the exocrine and endocrine compartments of the pancreas.

All text supplied by the authors. 